\title{
L'autobiographie comme conversion esthétique : les derniers écrits
}

\section{de Gabrielle Roy}

\section{Ginette Michaud}

\section{Abstract}

Gabrielle Roy's Last Writings. Autobiography as Aesthetic Conversion.

In 1975, Gabrielle Roy turned to an autobiographical project, worthy of being read as a major literary work rather than as an exemplar of Quebec literature-albeit its fate has been seen as subject for commentary rather than works.

\section{Citer ce document / Cite this document :}

Michaud Ginette. L'autobiographie comme conversion esthétique : les derniers écrits de Gabrielle Roy. In: Littérature, $\mathrm{n}^{\circ} 113$ 1999. La littérature, au Québec. pp. 95-114;

doi : $10.3406 /$ litt.1999.1617

http://www.persee.fr/doc/litt_0047-4800_1999_num_113_1_1617

Document généré le 01/06/2016 


\title{
L'autobiographie comme conversion esthétique*
}

\section{les derniers écrits de Gabrielle Roy}

\begin{abstract}
Je peux parler d'elle sans gêne. Cette enfant que je fus m'est aussi étrangère que j'aurais pu l'être à ses yeux si seulement ce soir-là, à Porée de la vie comme on dit, elle avait pu m'apercevoir telle que je suis aujourd'hui. De la naissance à la mort, de la mort à la naissance, nous ne cessons par le souvenir, par le rêve, d'aller comme l'une vers l'autre, à notre propre rencontre, alors que croît entre nous la distance.
\end{abstract}

Gabrielle Roy, la Détresse et l'Enchantement, p. 80 .

rançois Ricard formulait il y a quelque temps une hypothèse intéressante au sujet de l'œuvre de Gabrielle Roy, l'élisant, «pour qui étudie l'autobiographie littéraire », comme un objet d'étude tout à fait privilégié, dont l'intérêt dépassait largement, précisait-il, « le seul cadre de la littérature québécoise et canadienne (1) ». Quiconque a lu l'œuvre de Roy et tout particulièrement ses derniers écrits ne peut que souscrire à cette observation, et je voudrais à mon tour faire écho à cette hypothèse en soulignant quelques-uns des traits qui ont fait de l'écriture autobiographique une aussi exemplaire démarche, où l'écrivain approfondit avec une rare lucidité le transfert complexe qui se produit entre la «vie » et sa « conversion esthétique » dès lors qu'on se retourne sur elle pour en tirer un sens.

Quand autour de 1975 Gabrielle Roy entreprend d'écrire son autobiographie - son autobiographie et non ses mémoires : il en va d'entrée de jeu pour elle d'une distinction fondamentale qui touche la qualité même de l'écriture investie dans ce projet -, elle accomplit sans doute la plus extraordinaire conversion de son parcours d'écrivain, un parcours qui a pourtant

* Ce texte est la version remaniée d'une conférence donnée à l'université Harvard en avril 1998. grapbiques de la francophonie, Actes du colloque de Bordeaux 21, 22 et 23 mai 1994, textes réunis et présentés par Martine Mathieu, Paris, CELFA/'Harmattan, 1996, p. 23. 
été marqué par une série de réorientations décisives, de métamorphoses (2) même, comme l'a bien vu François Ricard, son biographe et plus éclairé critique. Signalons-en quelques-unes parmi les plus spectaculaires : d'abord, l'abandon du style réaliste après Bonbeur d'occasion pour le style «idyllique » de La Petite Poule d'eau, qui rompt violemment avec toutes les attentes de la critique ; ensuite, le déplacement d'un roman de type biographique fortement structuré par des effets de contrastes et de symétries à un récit plus libre dans sa forme et pris en voix par un « je » relevant de plus en plus du mode autobiographique ; la substitution, enfin, du personnage de la fille comme narratrice à celui de la mère, autre point tournant capital dans une œuvre tout imprégnée par ces relations mère-fille (ce changement narratif particulièrement significatif advient avec La Route d'Altamont, en 1966).

Cette série de mutations esthétiques nous indique que ce n'est pas la première fois que Gabrielle Roy se retrouve à la croisée des chemins, une métaphore qui traverse d'ailleurs toute La Détresse et l'Enchantement et que nous retrouverons également en ouverture du Temps qui m'a manqué. Lorsque vieille dame déjà Gabrielle Roy tente, dans les années qui précèdent sa mort en 1983, de ressaisir sa vie dans un récit de soi, qui doit autant à l'autobiographie classique qu'à certains dispositifs de l'autofiction, elle amorce une conversion autrement plus significative qui modifiera en retour profondément notre perception de son œuvre. Car ce n'est certes pas le moindre paradoxe de cette entreprise que de représenter la période la plus riche de sa carrière d'écrivain : «En moins de dix ans, malgré son âge et la détérioration croissante de sa santé, non seulement elle publie de façon régulière, mais elle donne quelques-uns de ses plus beaux écrits : Un jardin au bout du monde (1975), Ces enfants de ma vie (1979), La Détresse et l'Enchantement (1984) [3]», auxquels il faut aujourd'hui ajouter l'opuscule ultime, Le Temps qui m'a manqué paru en 1997. L'écriture de Roy gagne dans ces textes autobiographiques toujours davantage en maîtrise, en dépouillement et en justesse de ton, comme si elle s'épurait avec chaque nouvelle œuvre mémorielle, allant toujours plus à l'essentiel et abordant enfin à la toute fin le cour, le noyau embryonnaire sur lequel s'était édifiée l'architecture complexe de toute l'œuvre. On pensera ici à la célèbre métaphore proustienne qui fait également «porter sans fléchir, sur [une] gouttelette presque impalpable, l'édifice immense du souvenir (4) ».

Cette dernière période de la vie de Gabrielle Roy est donc totalement vouée au «temps de la mémoire », qu'on pourrait précisément qualifier de «proustienne» si le modèle n'était trop écrasant, puisqu'elle «se déroule tout entière sous le signe de la remémoration et de l'imagination autobiogra- 
phique ». Quelque ambiguë que soit cette dernière expression, c'est bien celle qui décrit le mieux la visée de Gabrielle Roy. Il ne s'agit pas seulement pour elle dans l'écriture autobiographique de la reconstitution des souvenirs, anecdotes ou événements qui ont formé le cours de sa vie et influé sur son «destin » d'écrivain, il ne s'agit pas d'une simple reprise à des fins d'idéalisation de soi (bien que cette dimension édifiante n'est pas complètement absente de La Détresse..), mais bien d'une forme d'autoengendrement d'elle-même comme sujet écrivain, bref une phase particulièrement féconde de sa démarche par laquelle elle s'engage dans une œuvre elle-même de part en part création, au sens le plus élevé du terme. Il est clair que l'autobiographie ne se limite pas pour Roy à un « réservoir d'impressions et d'images (5) », mais qu'elle est posée comme «l'objet même de l'écriture: l'aveu, la recherche de soi et l'expression de ce qui doit être donné et reçu comme la vérité de l'être qui écrit (6) ».

La profondeur de cette construction de soi est d'ailleurs telle qu'on peut dire que c'est bien l'écriture autobiographique qui fera le plus pour remodeler notre compréhension de l'œuvre de Roy. Longtemps infiltré dans la fiction - André Brochu fera remarquer avec raison que «le ferment autobiographique travaill[ait] déjà au démembrement de l'organisation romanesque (7) » depuis longtemps, sans doute dès La Petite Poule d'eau et même le très réaliste Bonbeur d'occasion, jusqu'aux récits toujours plus intimistes de Rue Deschambault, de La Mortagne secrète et de La Route d'Altamont - le récit de soi qu'elle entreprend dans La Détresse et l'Enchantement est d'une tout autre envergure et suscitera de nombreuses relectures, notamment d'orientation féministe, qui feront beaucoup pour remettre en question le bien-fondé de la vision traditionaliste qui pesait toujours sur l'œuvre de Roy, plus souvent jugée en des termes humanistes qu'esthétiques : ces travaux - je pense surtout à ceux de Patricia Smart, de Nicole Bourbonnais, d'Agnès Whitfield, et surtout de Lori Saint-Martin - nous permettront d'avoir un point de vue beaucoup plus nuancé sur la représentation des personnages féminins, et tout particulièrement des mères et de leurs filles, de leurs relations ambivalentes, tendues mais créatrices, dans l'univers romanesque de Roy. En outre, certains thèmes majeurs de l'œuvre fictionnelle - dont celui des rapports mère/fille - ne trouveront leur pleine élaboration que dans l'autobiographie, et tout particulièrement dans ce texte des adieux qu'est Le Temps qui m'a manqué. Je reviendrai sur ce travail de deuil qui est aussi travail d'écriture, mais soulignons pour l'instant l'impact de l'autobiographie sur toute l'œuvre, qu'elle aura, d'une certaine manière, libérée en engageant à la relire d'un autre angle, à partir de perspectives nouvelles, et surtout en la dégageant d'une forme d'image-écran qui 
en avait figé l'interprétation. La publication posthume, selon les volontés de l'écrivain, de La Détresse et l'Enchantement en 1984 et de sa suite en 1997, de même que la parution en 1996 de la monumentale biographie de François Ricard (8), ont donc introduit un nouvel équilibre à l'intérieur même de l'œuvre en mettant en évidence la position symétrique, à trente ans d'intervalle, de la fiction et de l'autobiographie : la succession des récits autobiographiques apparaît désormais, comme le note Brochu, «comme le digne pendant du roman qui avait servi de point de départ à l'aventure d'écrire (9) ».

Par ailleurs, le passage à l'écriture autobiographique permet également de mieux mesurer l'originalité de Roy comme écrivain : dans La Détresse et l'Enchantement, non seulement se met-elle à la recherche de ses propres sources, mais elle se fait attentive aux relations complexes entre création et mémoire, à «la redécouverte ou rédemption, par l'imaginaire, de la vie qui, vécue autrefois, ne l'a pas été pleinement avant d'être ainsi transformée en écriture (10) ». Ce qui fascine Roy dans ce projet c'est très exactement cela : cet incessant échange entre la vie et le récit, cette réversibilité réciproque et recommencée entre le matériau biographique et sa transmutation littéraire, autrement dit cette conversion esthétique qui ne cesse pas de se produire et qui, phénomène toujours étrange, va dans les deux sens, conversion qui rehausse la vie en œuvre et renvoie l'œuvre à la vie, altérant par cette recréation le passé et le souvenir eux-mêmes, qui ne cessent jamais de prendre forme et d'être transformés par le fantasme et l'imaginaire. Comme l'observe Ricard, «la vie, ici, ne fait pas que nourrir l'œuvre ou lui fournir les matériaux nécessaires à son édification. L'échange inverse se produit également, et prend peut-être même une importance plus grande encore : littéralement, l'œuvre construit, ou reconstruit (et j'ajouterai : déconstruit), la biographie de l'écrivain, et elle trouve dans cette construction-là une de ses fonctions essentielles $(11) \gg$.

\section{ENTRE-DEUX : DE L'AUTOBIOGRAPHIE CLASSIQUE A L'AUTOFICTION}

Sur le plan de l'écriture proprement dite, souvent célébrée pour les qualités de son style limpide (ces qualificatifs reviennent souvent sous la plume des critiques : pureté, transparence, fluidité), Gabrielle Roy se trouve

8 Cette biographie, conçue comme une « réponse » à l'ouvre, instaure en retour un rapport dialogique assez exceptionnel avec elle. Indispensable contrepoint du texte autobiographique, cette biographie corrige sur des points précis l'image de soi trop lénifiante que Roy avait construite, mais surtout elle marque l'écart entre la «vie» et son esthétisation, souvent synonyme d'embellissement et d'idéalisation dans l'autobiographie. Pierre Nepveu note aussi le «contraste sassissant » entre, d'une part, le classicisme de l'écriture et Pharmonie des rapports humains rayonnant dans les derniers livres de Roy et, d'autre part, le « vécu tendu et angoissé qui a été leur creuset » («L'écriture souveraine, malgré tout », Spirale, n 151, novembre-décembre 1996, p. 8).

9 André Brochu, art. cité, p. 216. 
déportée avec l'écriture autobiographique sur un tout autre terrain, où d'autres traits plutôt caractéristiques de la modernité viennent à l'avantplan : fragmentation, inachèvement, discontinuité, fragilité de l'identité, autoréflexivité. De fait, le projet autobiographique de Roy, s'il n'adhère pas totalement aux jeux spéculaires de l'autofiction, prend résolument ses distances à l'endroit de l'autobiographie classique. Rappelons brièvement quelques éléments de la définition canonique du genre : un récit vrai, souvent discrédité justement sur le plan aléthique, autocentré sur un sujet au narcissisme envahissant, qui institue un pacte en son nom propre, qui lie auteur, narrateur et personnage: son mode discursif est, comme l'a noté avec finesse Marie Darrieussecq, l'assertion, mais l'autobiographie, loin d'être un acte de langage simple comme on a eu souvent tendance à la réduire, double cette affirmation d'une injonction adressée au lecteur qu'on peut traduire par un « croyez que (12)». On pense irrésistiblement à ces incessantes demandes de confiance, à toutes ces autojustifications et appels réitérés de l'autobiographie rousseauiste, qui reste le texte fondateur de la tradition. Chez Gabrielle Roy, la demande de croyance et l'autojustification se font sans doute plus discrètes que chez Rousseau, mais elles soutiennent néanmoins l'entreprise du dire : « Toujours est-il que je disais - car il faut toujours fournir aux autres une explication plausible de nos actes - $[. ..] \gg(D E$, 182). Par opposition, l'autofiction est, paradoxalement, plus simple, en tant qu'acte de langage tout au moins : elle consiste à «feindre une assertion autobiographique (13) » et remplace la demande de croyance par un «imaginez que (14) », car l'autofiction assume, comme on le sait, de manière délibérée l'«impossible réduction de l'autobiographie à l'énoncé de réalité (15)»; elle demande à la fois «à être crue et demande à être non crue (16) », jouant sur les deux tableaux - le factuel et le fictif - en même temps, assumant volontairement " "cette impossible sincérite" ou objectivité, et intégr[ant] la part de brouillage et de fiction due en particulier à l'inconscient (17)».

Seule l'écriture, non le souvenir qui en est seulement le support, ouvre le passage vers une vérité du sujet, aussi insaisissable soit-elle. Il y a donc bien aussi dans cette écriture autobiographique " "fictionnalisation" du factuel et "factualisation" du fictif (18)», ce qui rend à certains moments le texte de Roy indécidable et le rapproche de l'autofiction, paradoxalement plus franche parce qu'elle sait qu'elle ment et invente jusqu'à un certain point le souvenir. Je renverrai à ce propos à l'ouverture, assez remarquable sous ce rapport, de La Détresse..., où la narratrice va fonder en vérité toute son entreprise. Soulignons l'audace de cette scène inaugurale où la narratrice, jouant de deux voix (elle est à la fois représentée sous les traits de 
l'enfant de neuf ans et de l'adulte réfléchissant rétrospectivement sur ellemême), va élire comme point d'origine de sa propre conscience le récit mi-vrai mi-fantasmé de sa mère, un récit qui est lui-même, sur le plan esthétique, fragmentaire, décousu et troué de blancs de mémoire. C'est le tressage du vécu et du fictif, le mélange du réel et de l'imaginaire qui fascinera toujours au premier chef Gabrielle Roy.

D'autres traits éloignent encore ces œuvres de Roy de la stricte autobiographie classique. D'abord, le fait qu'elles aient été pensées d'avance comme posthumes, c'est-à-dire comme un legs testamentaire porteur du sens de l'œuvre, et peut-être même, c'est ce que nous verrons dans Le Temps qui 'm'a manqué, de son secret (le secret n'a pas à être défini de manière réductrice comme ce que l'on cache à l'autre, mais apparaît plutôt comme le signe insu de ce qui se dérobe à notre propre saisie). Ce n'est pas tant le fait que cette autobiographie soit posthume qui frappe ici le lecteur (toute autobiographique relève d'un tel dispositif testamentaire et se déploie, à des degrés divers, comme thanatographie), mais l'adresse radicale à l'autre qui la fonde. Autant, comme le dit Serge Doubrovsky, «l'autobiographie classique croit à la parthénogenèse scripturale : le sujet y naît d'un seul. Regard de soi sur soi, récit de soi par soi, le même y naît toujours du même (19) », autant l'écriture autobiographique chez Gabrielle Roy passe nécessairement par le rapport à l'autre : l'auto-connaissance du sujet y est inséparable de la reconnaissance de l'autre, et ce n'est certes pas un hasard si la scène inaugurale de La Détresse... met l'accent sur le récit généalogique de la mère - un récit qui a d'ailleurs pour objet la filiation phylogénétique, qui dépasse donc l'histoire du seul individu pour l'inscrire d'emblée dans une lignée (20) -, récit écouté par la fille et posé comme le fondement de sa conscience. Ce rapport à l'autre est éminemment révélateur d'une position fondamentale de Roy comme écrivain et détourne déjà en lui-même l'auto-affectation ou le narcissisme de l'autobiographie classique.

Ce rapport à l'autre est encore souligné à l'autre extrémité de l'œuvre autobiographique dans l'ultime scène du Temps qui m'a manqué alors que, la mère morte, il s'agit par le travail de deuil qu'est l'écriture de s'adresser désormais à l'autre en soi. Gabrielle Roy décrit en des termes percutants le transfert qui se produit alors qu'elle introjecte en elle la voix de sa mère: «C'est en cette nuit de juin 1943, quelque part dans une forêt de l'Ontario,

19 Serge Doubrovsky, «Autobiographie/vérité/psychanalyse », dans Autobiographiques : de Corneille à Sartre, Paris, PUF, 1988, p. 72 .

20 On sait que cette dimension phylogénétique, ou tout au moins historique, est fortement mise en relief dans l'incipit et la clausule de La Détresse... : le récit troué dont la mère sait seulement des «bouts», «transmis de génération en génération ", où reviennent les figures des ancêtres consolés par ce récit mémoriel qui les fait revivre, constitue l'un des traits les plus originaux de cette autobiographie. La structure circulaire du texte où elle apparait à la fois comme la question et la réponse éclairant la vie individuelle de ce sujet particulier confirme son importance. La mythographie personnelle de l'écrivain est ici étroitement liée, par ce récit de la mère (un récit troué, lacunaire, mais néanmoins inlassablement récité et transmis), au son de son "espèce » 
que commença entre ma mère et moi le singulier échange de voix où c'est pourtant moi seule qui reçois ses confidences à travers le silence [...] (21) ». Déjà donc on pourrait dire de l'autoportrait de Gabrielle Roy qu'il n'est toujours qu'un « hétéroportrait (22)» une «auto-connaissance à deux», où « l'un y naît de deux, le même y advient du même et de l'autre (23) », d'où ces constants allers et retours, mouvements d'éloignement et de rapprochement, de fort/da qui marquent tout particulièrement le rapport à la mère chez Roy, mais aussi sur le plan psychique le mouvement même de la pensée et de l'écriture (24).

Autre aspect qui démarque l'écriture de Roy de l'autobiographie classique: le renoncement à toute exhaustivité et même à toute exactitude quant aux faits dits objectifs rapportés. Ce renoncement a aussi des conséquences esthétiques, comme en témoigne ce passage où elle récuse le réalisme dont elle avait pourtant usé comme romancière :

Mais nulle part je ne me suis attachée à le décrire absolument ressemblant. C'est une tâche dont je pense être incapable maintenant. Il me faut dissocier les éléments, les rassembler, en écarter, ajouter, délaisser, inventer peut-être, jeu par lequel j'arrive parfois à faire passer le ton le plus vrai, qui n'est dans aucun détail précis ni même dans l'ensemble, mais quelque part dans le bizarre assemblage, presque aussi insaisissable lui-même que l'insaisissable essentiel auquel je donne la chasse. Décrire fidèlement une maison telle que sous mes yeux, ou une rue ou un petit bistrot de coin comme je l'ai fait dans Bonbeur d'occasion, à présent m'ennuierait mortellement. Je m'y astreignais, alors, par souci de réalisme, il est vrai, mais aussi pour retenir une imagination trop débordante et me contraindre à bien examiner toutes choses pour ne pas glisser à la paresse de décrire sans fondement sûr $(D E, 112)$.

Si l'esthétique réaliste est désormais incompatible avec l'écriture autobiographique, celle-ci ne peut davantage se plier aux autres conventions du récit mimétique, en particulier à ce qui a trait à l'ordre du récit, à son déploiement sur l'axe du temps. La Détresse et l'Enchantement ne commence pas avec le canonique «Je suis né le... », il escamote même toute la période de la première enfance (qui ne peut être racontée par le sujet même, mais seulement reconstituée à travers le récit que les adultes lui transmettent).

21 Gabrielle Roy, Le Temps qui m'a manqué, édition préparée par François Ricard, Dominique Fortier et Jane Everett, Montréal, Boréal, «Cahiers Gabrielle Roy », 1997, p. 25. Désormais désigné par le signe TM, suivi de la page. 22 Serge Doubrovsky, op. cil., p. 73.

$23 \mathrm{Ibid}$, p. 72. On s'approche ici de l'expérience de la psychanalyse, « puisque c'est de l'écoute de l'autre que la vérité revient (advient) dans le discours où le sujet tâche à se saisir » (ibid., p. 65).

24 Lori Saint-Martin a très bien montré comment la mère imprime aux textes de Roy leur forme si particulière, qu'il s'agisse de structure narrative, de réseaux métaphoriques, de tournures syntaxiques, de marques de l'énonciation ou de figures rhétoriques («Gabrielle Roy: écriture et réparation », dans Le Nom de la mère. Maternité et textualité dans l'écriture des femmes québécoises, Québec, Nota bene, à paraître en 1999). 
Le récit de vie est donc nécessairement incomplet, saisissant la narratrice pour la première fois à neuf ans et la laissant dans la scène finale du Temps qui m'a manqué à trente-trois ans, au seuil de l'écriture qui la mènera trois ans plus tard à Bonbeur d'occasion. En outre, Gabrielle Roy ne respecte pas scrupuleusement la linéarité chronologique, même si dans La Détresse... on continue de suivre le fil du temps (« [...] de ces tâtonnements, de ces allers, de ces errances, $j$ 'ai appris comme je n'aurais appris d'aucune ligne droite que $j$ 'aurais suivie par simple opiniâtreté. [...] Longtemps j'ai voyagé sans boussole. Mais aussi pour la traversée de la vie, que vaut une boussole? », $D E, 299-300)$ : son récit est complexe sur le plan du déroulement temporel, ponctué de boucles, d'analepses et de ruptures et, sous ses apparences de chronique, l'histoire racontée est beaucoup moins linéaire qu'elle ne fonctionne en réalité par «images brèves et détachées», «collage d'instants, d'aperçus, de morceaux de vie dont la position sur l'axe du temps garde toujours une large mesure d'imprécision, comme si le récit suivi était impraticable (25) ». Le parcours ainsi délimité souscrit donc d'emblée à un découpage où il ne s'agit pas, à l'évidence, de tout raconter, mais de se concentrer sur les années de formation, de restituer les conditions et les événements d'un Bildungsroman intellectuel et l'émergence de sa sensibilité, de son rapport propre au monde et aux choses préparant sa naissance comme écrivain. Or, il y a déjà, dans ce choix, une ruse remarquable : Gabrielle Roy comble par cette œuvre autobiographique la période où elle n'écrit pas, où elle est à toutes fins utiles hors de l'espace d'écriture : elle écrit son autobiographie pour masquer le fait qu'elle n'a pas été dans l'écriture dès l'origine, pour faire oublier qu'il y eut une vie avant l'écriture, précisément en l'écrivant, cette vie, en la transfigurant. D'ailleurs, comme l'ont fait remarquer plusieurs commentateurs, d'une certaine façon, il n'y aurait plus, après la fin de La Détresse... où elle écrit à sa mère pour l'informer de sa double décision de ne pas rentrer au Manitoba et de «plong[er] dans l'écriture, moi qui tout à coup percevais combien peu je savais encore m'y prendre » $(D E, 504)$, rien à écrire... que l'œuvre elle-même (26), et cette fin est encore plus vraie dans Le Temps qui m'a manqué où, pour supporter la douleur de la perte de sa mère, elle se jette à corps perdu dans l'écriture (27).

25 François Ricard, op. cit., p. 38.

26 Cette structure circulaire fait évidemment penser à la Recherche proustienne, au reploiement infini de la fin en commencement du texte que nous sommes en train de lire.

27 Il est intéressant de souligner que ce recours à l'écriture répète la réaction qu'elle dit avoir éprouvée à la mort de son père, alors qu'un désir, tout à fait intempestif dans le contexte de reconstituer la fameuse scène du bal chez le gouverneur - et tout particulièrement de décrire dans le moindre détail la toilette de sa mère déplace (vient à la place) de l'affect qu'elle ne ressent pas à ce moment : «Il me paraissait important d'assembler tous les éléments de cette histoire comme si c'était sa dernière chance [...] » $(D E, 99)$. Le récit répond donc, à ce moment comme lors de la mort de la mère, à un irrépressible désir de colmater la brèche ainsi ouverte, de suturer et de coudre ce qui a été troué par la mort. 


\section{"LE TEMPS QUI M'A MANQUE " : CONTREPOINT ET CONTRE-LECTURE}

Venons-en maintenant au Temps qui m'a manqué, ce texte ultime qui présente un statut assez particulier. Se donnant pour la suite de La Détresse et l'Enchantement - et ce lien est fortement souligné sur les plans stylistique et temporel par l'adverbe «longtemps» de l'incipit («Longtemps il m'avait semblé que les rails ne me chanteraient jamais autre chose que le bonheur » $T M, 13$ ), qui amorce la réponse longtemps différée à la question ouvrant La Détresse... : «Quand donc ai-je pris conscience ?...»-, cette suite fut pourtant publiée beaucoup plus tard, treize ans après l'œuvre à laquelle elle se rattache. Cet appendice (c'est le début de la troisième partie, alors que l'autobiographie devait en compter quatre au total) paraîtra donc à part, comme un morceau détaché, opuscule autonome en lui-même. À la fois fragment et totalité, et à cause de sa finalité, ce texte occupe donc une place tout à fait privilégiée dans l'œuvre, en offrant une ponctuation particulièrement forte et singulière : même s'il y aura sans doute d'autres inédits de Roy qui seront éventuellement édités, celui-ci restera en effet pour toujours le dernier texte écrit de sa main, celui qu'elle aura « achevé », avant d'être trop malade pour écrire (28). Comme le titre le suggère, le manuscrit a sans doute été interrompu par la mort de l'écrivain, survenue en 1983 (29), mais « le temps qui a manqué » renvoie tout aussi bien à la mort qui a emporté trop vite sa mère en juin 1943 avant que Gabrielle n'ait pu la revoir. Le fragment que nous lisons - la troisième version du manuscrit, qui n'était vraisemblablement pas la dernière - est donc à la fois inachevé (quelques phrases laissées en friche sont en effet un peu moins harmonieuses que d'habitude) et pourtant très achevé : ce bref récit, même interrompu, se rend en effet jusqu'au bout de lui-même et met exemplairement en scène une fin qui se révèle commencement. En ce sens, il répète structurellement la fin de La Détresse... où, renonçant à répondre aux appels pressants de sa mère, la narratrice choisissait de rester à Montréal et d'écrire « en ce soir du commencement » $(D E, 504)$. Ces pages continuent donc, du moins en apparence, là où La Détresse... s'était arrêtée, avec le refus de revenir au Manitoba et sa décision de se lancer dans l'écriture. De fait, « la narratrice y relate ses débuts comme reporter [...], sa découverte de Montréal et du Québec, ses efforts pour écrire, la genèse de son premier roman (30) », mais toutes ces choses de la vie courante sont éclipsées par la gravité d'un événement d'une portée incommensurable, qui introduit tout de suite dans le

28 Dans le film de Léa Pool (Gabrielle Roy. Les chemins de l'écriture, ONF, 1998), la meilleure amie de Gabrielle Roy, Berthe Simard, confirmait que l'écriture de ce dernier fragment autobiographique avait été très difficile pour elle, au point qu' elle se demandait si elle aurait l'énergie nécessaire pour le mener à terme.

29 La narratrice précise elle-même qu'elle a soixante-douze ans au moment où elle écrit ces pages : « Le même but vers lequel je cours encore à près de soixante-douze ans et dont je me demanderai sans doute si j'en ai le temps avant de mourir quel il était » $(T M, 19)$. Gabrielle Roy mourra deux ans plus tard, à soixante-quatorze ans. 30 «Avertissement des éditeurs», dans Le Temps qui m'a manqué, p. 10. 
récit la rupture la plus ébranlante qui soit : la mort de la mère. D'une certaine manière, il n'est pas exagéré de suggérer que toute l'œuvre de Gabrielle Roy, tous les chemins d'écriture, aussi divers aient-ils été, ont été empruntés pour aboutir à ce récit-limite, ce récit qui est à la fois sans suite possible et le noyau, l'embryon qui engendre à rebours toute l'œuvre. Autrement dit, tout ce qui précède aura été une manière de gagner du temps pour pouvoir s'approcher enfin de l'indicible de cette scène qui donnera, en retour, naissance à l'œuvre. En ce sens, Le Temps qui m'a manqué est bien un fragment plus grand que le tout : cette scène de la mort de la mère, l'écriture du deuil qui en rend compte marquent aussi la naissance de l'écrivain se mettant en scène dans un extraordinaire auto-engendrement, réalisant ainsi son ultime conversion esthétique.

La question du temps s'éclaire ici encore. Il fallait que la narratrice approche de sa propre fin pour vraiment ressaisir le deuil de sa mère. Comme elle l'écrit dans La Détresse... - et en un sens, toute autobiographie vaut essentiellement en tant que conception singulière du temps -, pour Gabrielle Roy, les temps doivent se rejoindre, se superposer, pour être vraiment éprouvés et pouvoir s'écrire :

Maman, à cette époque, allait sur ses soixante-sept ou soixante-huit ans. L'âge que j'ai maintenant, alors que je prends le temps enfin de m'interroger sur ce qu'elle a pu ressentir d'infini chagrin. Tout cela est bien curieux. Il semblerait que l'on ne rejoint vraiment ses gens que lorsqu'on atteint l'âge qu'ils avaient alors qu'à côté d'eux, on ne comprenait rien à leur vaste solitude ( $D E, 143$. Je souligne).

On sent bien dans cette phrase le croisement tragique, l'incommunicabilité entre les êtres qui ne peuvent jamais se rejoindre que trop tard (3i).

La question du temps, du travail du temps, est donc au cœur de ce récit qui commence par l'adverbe proustien par excellence, «longtemps», et qui institue une circularité, une réversibilité de la fin comme commencement qui ne peut pas ne pas faire penser à la Recherche. (Ce n'est pas le seul rapprochement qu'on pourrait esquisser : tout comme Proust ne peut commencer la Recherche qu'après la mort de sa mère, Gabrielle Roy fonde avec ce dernier récit, tout au moins sur le plan symbolique, sa venue à l'écriture sur la mort de la sienne. Dans les deux cas, l'œuvre comme travail de deuil est une composante fondatrice de leur entreprise.)

Mais retraçons rapidement les grandes lignes du récit, divisé en quatre séquences, avant de nous arrêter un peu plus longuement sur la toute dernière scène. Dans la première partie, la narratrice est dans le train, en route vers le Manitoba pour les funérailles de sa mère. Bercées par le rythme du 
train, ses pensées suivent divers aiguillages, mais reviennent toujours à celle qui troue impitoyablement le récit : « Maman est morte. » Parmi les associations qui lui reviennent en mémoire, Gabrielle évoque dans une longue analepse «la maison où était née maman »: «Tout ici me semblait correspondre à la description qu'elle en avait cent fois reprise. Je ne pense pas l'avoir vue aussi nettement que ce jour-là, alors qu'à mes yeux n'apparaissait rien d'autre que de la neige poudrante entre des épinettes figées. [...] et en un sens les collines étaient encore plus en moi par la mémoire que par mon regard qui en saisissait le doux profil bleuté au-delà de la plaine de neige » (TM, 22-23). Ce passage est important parce que ce retour au pays natal de la mère préfigure déjà la régression déclenchée par sa mort et qui culminera dans la dernière séquence du récit. On y voit aussi une première formulation du legs esthétique de la mère conteuse : reprenant cent fois la description (le mot « reprise» est plus suggestif, inscrivant le motif de la couture et du reprisage pratiqués par la mère), la mère transmet toute une poétique du récit qui sera recueilli par sa fille, pour qui la mémoire, c'est-à-dire la recréation, l'invention, compte plus que «son regard», c'est-à-dire la réalité. En outre, ce passage nous frappe parce que la fille y réitère une attitude fondamentale face à la mère: non seulement est-elle sûre d'avoir retrouvé la « vraie » maison au hasard de sa randonnée, mais elle s'empresse d'en faire à son tour la description à sa mère dans une lettre, « m'attachant surtout à rendre son silence émouvant et la teinte du ciel, d'un mauve délicat, audessus de la crête d'où, enfant, elle avait dû venir regarder le monde, la vie, et tâcher de percevoir ce qu'elle deviendrait (32)» (TM, 23-24). On voit ici, de la description de la mère à celle de la fille, qui l'imagine enfant et l'enfante en réalité dans l'écriture, le « singulier échange de voix » qui s'instaure dans un complexe aller et retour entre la mère conteuse et la fille écrivain qui lui donne voix. Elle reprend ici dans une autre tonalité l'idée déjà formulée dans La Détresse..., à savoir qu'elle écrira pour venger la mère et qu'elle lui offrira ses cadeaux-récits comme autant de médailles (33), bref,

32 On retrouve ici l'écho de son propre autoportrait en $\alpha$ cette inconnue de moi-même que je serai un jour » (cité par Ricard, op. cil., p. 99). Ce renversement des rôles, où la fille imagine la mère enfant à sa propre image, est significatif de l'échange des voix mère/fille qui se produit souvent dans l'œuvre de Roy. Voir, sur cette question, Lori Saint-Martin, «Mère et monde chez Gabrielle Roy», dans L'Autre Lecture. La crilique au Jéminin et les textes québécois, sous la direction de Lori Saint-Martin, t. 1, Montréal, XYZ éditeur, 1992.

33 « $[\ldots]$ elle était éblouie par mon tiroir plein de grosses médailles, et je la soupçonne de l'avoir souvent ouvert quand elle était seule pour les admirer à son aise $\gg(D E, 69)$ : on voit dans ce fantasme de la fille qu'elle cherche à soutenir le narcissisme défaillant de la mère par ces médailles-miroir où elle peut se mirer/s'admirer «à son aise » quand elle est seule, en secret. Roy revient encore dans Le Temps qui m'a manqué sur ces fameuses médailles : « $[\ldots]$ je me surpris à lui promettre, tout en pleurant, que j'obtiendrais pour la consoler et la rehausser, bien d'autres médailles, des dizaines de médailles. Ce que jai fait au reste, à en avoir encore un tiroir plein » (TM, 54. Je souligne). Mais cette promesse tenue ne fait pas le poids en comparaison de ce qu'elle n'a pas su donner à temps, notamment de n'avoir pas fait ses adieux à la mère et d'avoir ainsi transgressé une loi du devoir filial, comme le lui reproche Esther, figure substitut idéalisée de la mère : « $\AA$ travers le roulement du train j'entendis Esther, à la voix déformée par le téléphone, me lancer, de la cabine d'Úpshire: - Il est important d'aller dire adieu à ceux qui vont nous devancer dans la mort. -.. Mais si nous n'arrivons pas à temps !... Elle ne répondait pas. La ligne entre nous deux devait être coupée » (TM, 17). La non-réponse d'Esther, la ligne coupée sont en elles-mêmes la sanction d'un devoir aux morts (par extension aux ancêtres) qui n'aura pas été rendu. 
qu'elle deviendra, comme l'a noté Lori Saint-Martin avec perspicacité, son «trophée brillant (34)». Cette représentation de soi est trop idéaliste et altruiste pour ne pas être suspecte, car si Gabrielle choisit de devenir écrivain soi-disant pour soutenir la mère à sa manière (35) et la consoler de sa vie difficile, c'est bien tout autant pour s'en séparer et la perdre de vue, comme elle en a fait plus lucidement l'aveu dans cette vraie scène des adieux qui rend un son plus juste :

Je la vis serrer sur elle son manteau un peu étroit d'un geste que je reconnus seulement à cette minute lui avoir vu faire cent fois au moins et qui la peignait si bien telle qu'elle était, à la fois timide et fière. Elle me suivait de ses yeux éteints comme s'ils n'allaient cependant jamais me perdre - où j'irai ! - au bout de leur regard. L'expression m'en devint insoutenable. J'y voyais trop bien qu'elle voyait que je ne reviendrais pas. Que le sort me happait pour une tout autre vie. Le cœur me manqua. Car j'y saisis tout au fond, que je ne partais pas pour la venger, comme j'avais tellement aimé le croire, mais, mon Dieu, n'était-ce pas plutôt pour la perdre enfin de vue? [...]

Est-ce que je n'ai pas lu alors dans mon coeur le désir que j'avais peut-être toujours eu de m'échapper, de rompre avec la chaîne, avec mon pauvre peuple dépossédé ? Qui de nous ne l'a un jour souhaité ? Une si difficile fidélité !

Ensuite, je pense avoir versé des larmes. De honte ? De compassion ? Je ne le saurai jamais. J'ai peut-être pleuré de l'amer sentiment de désertion $(D E$, 242-243).

On voit à la lecture de cette scène que Gabrielle Roy est consciente que le but désintéressé qu'elle se donne, désir de vengeance ou de réparation de la mère, recouvre en fait une part beaucoup plus sombre de son désir d'émancipation, qui consiste à rompre, au contraire de ce qu'elle dit dans sa fiction «sublimante » où elle « rehausse » son moi, le lien de dépendance et de quasi-symbiose (36) qui la lie à la mère, à se séparer d'elle, à s'en désolidariser, à s'en éloigner. Dans cette perspective, le fantasme de réparation est seulement l'envers de l'ingratitude et de la honte ressentie bien longtemps après cet abandon et ce reniement.

Dans la seconde séquence, Gabrielle Roy relate les funérailles, en deux temps, de sa mère. Dans un fragment important, elle s'attache avec une minutie cruelle à décrire l'encolure de la robe de la morte dans son cercueil, 
rappel d'un motif récurrent de La Détresse... (37) [il s'agit du col dont elle se demandait s'il avait été volé lors de son séjour à Paris] et qui insiste encore sur le motif de la couture comme métaphore du legs esthétique de la mère conteuse et couturière, «faufileuse » d'histoires : «L'ourlet de l'encolure avait été entamé à petits points assez bien tirés qui étaient devenus plus lâches, inégaux, presque gauches, pour s'arrêter court non loin du but » $(T M, 51)$. Cette image s'applique également en un autre transfert esthétique à la conduite du récit même, alors que la narratrice essaie à son tour « de coudre ensemble les mille bouts de la pauvre histoire » (TM, 18), pour ne pas perdre son « fil interrompu », ce qu'elle « redoute tellement » $(T M, 21)$. $\mathrm{Ce}$ motif du lien est encore mis en relief, au moment de fermer le cercueil, dans le «vieux chapelet », objet symbolique s'il en est, qu'on détache des mains de la mère pour « le donner en souvenir » $(T M, 55)$ à Gabrielle : « Je m'aperçus qu'il tenait à peine ensemble, partout prêt à se rompre, rafistolé çà et là avec du fil à coudre. - Mon Dieu ! me suis-je écriée, elle n'avait même plus de chapelet! Regarde, Dédette. Ce ne sont que des bouts épars! » $(T M, 55)$. La pulsion de déliaison atteint tout aussi bien les objets, les êtres que le récit lui-même (dans le troisième mouvement, cette déliaison, caractéristique de la pulsion de mort, relâchera aussi les liens la rattachant à ses sœurs, comme si, la mère morte, plus rien ne tenait ensemble cette famille [38]).

Il y a donc deux cérémonies des adieux, l'une sous le signe du rituel, du «drapé noir », du «ton d'incantation», des «condoléances apprises» (TM, 57), l'autre dans une petite « chapelle rustique », toute de simplicité et de naturel, avec « des brassées de fleurs de toutes sortes » éparpillées sur le cercueil, dans la beauté d'un jour de juin, sous «le haut ciel manitobain, peut-être plus beau que jamais pour être vu avec des yeux mouillés » (TM, 57-58). Cette seconde séquence se termine sur le regard extasié de la narratrice regardant «le vol plané des oiseaux blancs au cri de solitude » dont sa mère lui avait dit dans son enfance, en lui serrant avec force la main, que ce cri appelait «l'âme à la liberté »: «Je venais de comprendre que de ma mère morte je recevais, à travers ce qu'elle avait aimé et m'avait fait aimer, plus encore que ce qu'elle m'avait donné au temps que nous appelions celui de sa vie $(T M, 60)$. Ici encore, le motif du transfert, l'introjection de la

37 On pense à cette scène où la mère lui coud une robe de bal : « Ma robe ne fait pas très mode. L'ourlet du bas ondule quelque peu. L'encolure est un peu gauche aussi, comme si maman avait donné un coup de ciseau maladroit, qu'il avait été impossible de reprendre" $(D E, 79)$. Le même ourlet imparfait, la même encolure maladroite se répondent de loin en loin, pour souligner, comme dans les « pauvres» lettres à « l'écriture toute défaite » $(T M, 19)$ de la mère, écrites « au prix de Dieu sait quels efforts ( $(T M, 19)$, « envoyées inachevées sans peut-être qu'elle s'en fût aperçue » (TM, 29), un essentiel inachèvement, une usure, une décomposition qui inscrivent déjà en eux.mêmes la déliaison de la mort dans ces « pauvres » objets.

38 Virginia Woolf évoquera dans Instants de ma vie un effondrement analogue lors de la mort de sa mère : «Une vie commune de la famille très joyeuse, très agitée, regorgeant de monde; et ma mère en était le centre ; il était ma mère. On en eut la preuve le 5 mai 1895. Car, dès ce jour il n'en resta plus rien » (Paris, Stock, 1986 [1976], p. 97, cité par Maud Mannoni, Elles ne savent pas ce qu'elles disent, Paris, Denoël, « L'Espace analyti. que », 1998, p. 33). 
voix de la mère en elle - première phase du deuil — affleure, sous la force d'une conversion qui touche au sublime (il s'agit bien de rehausser la mère en soi).

Le troisième mouvement du texte amorce le temps de l'« après », alors que Gabrielle parle avec ses sœurs de la mère disparue, mais sans pouvoir partager avec elles son image intérieure :

Je les écoutais avec la plus vive stupeur. Pas plus que je ne pouvais voir ma mère dans la femme qu'elles avaient connue, pas plus sans doute n'auraientelles pu la reconnaître dans l'être imaginatif que je chérissais [...]. Je renonçais à leur faire comprendre notre mère telle qu'elle me paraissait avoir été. Une pesante tristesse me venait alors, comme si j'en étais à la voir mourir une deuxième fois, et cette fois irrévocablement $(T M, 65)$.

C'est précisément cette dissolution de l'image de la mère qui « remet sur le chantier le travail du deuil, de l'innommable pour accoucher du don d'une cuvre qui se détache (39) » d'elle dans le dernier mouvement du texte. C'est en effet à partir de cette deuxième perte que le travail de deuil commence vraiment à faire son œuvre. Après avoir revisité les lieux privilégiés de son enfance - le « flot lent de la rivière », «l'herbe rugueuse », cette «brousse étrange», ce «milieu sauvage sur la pointe des buissons » (TM, 66) et tout particulièrement le petit bois de chênes noirs, «transplanté intact » $(T M, 67)$ - comme pour s'en séparer définitivement, incapable de surmonter la distance qui l'éloigne de ses sœurs, «devant leur mal trop pareil au mien que je sentais parfois vouloir renaitre à leur contact » (TM, 73), elle prend la fuite.

Nous la retrouvons dans le dernier fragment en Gaspésie où elle trouve refuge à Port-Daniel, au bord de la mer, dans une pension comme elle en a l'habitude depuis Upshire et surtout une chambre à soi (40), comme Virginia Woolf - et pas n'importe quelle chambre : elle demande une « chambre dans laquelle la mer entre de tous côtés» $(T M, 84):$ inutile d'insister sur la permutation constante qu'autorise ce signifiant et le « grand

39 Maud Mannoni, op. cit., p. 54. «Du vide qui s'est créé s'élève un appel, comme pour renouer avec l'enfant perdu en elle, enfant à la recherche d'un écho, d'une "contre-voix" maternelle », note Maud Manonni à propos de Virginia Woolf (ibid., p. 55). La remarque vaut également pour Gabrielle Roy.

40 On trouve souvent chez Roy un éloge des «chambres à soi » digne de Virginia Woolf : «Ah, la douce chambre, aussi accueillante sinon encore plus que celle de Matravers et même de Century Cottage. Ai-je été chanceuse, quand j'y pense, d'avoir eu pour y dormir, rêver, réfléchir, de pareilles chambres déjà si pleines de songerie en suspens. Sans eau courante, sans cabinet de toilette même sans électricité, au fond sans commodité aucune, elle eut toute mon amitié dès le premier regard, avec ses jolis tapis crochetés jetés çà et là sur le plancher fait de larges planches peintes couleur du soleil, avec sa commode ancienne, son ample chaise berçante et surtout deux fenêtres à fins rideaux par lesquelles entrait en biais et de façade toute la baie de Port-Daniel, éblouissante de lumière " $(T M, 85)$. Sur ce rôle des fenêtres dans la fiction de Woolf, fenêtres également si présentes dans toutes les chambres de Roy, Maud Mannoni observe avec perspicacité que cette fenêtre est « déjà un regard. Du moment où ce regard existe, Lily peut se sentir objet de désir de l'Autre », citant Lacan : «L'essentiel n'est pas ce qui est là, ce qui est vu. Ce qui la structure, c'est ce qui n'est pas là » 
désir d'eau (41) » lié à la mère que cette mer amniotique représente. C'est dans cette « chambre des merveilles » $(T M, 85-86)$, qui ouvre sur une nature parfaite où elle retrouve « un monde encore si beau » $(T M, 87)$ où «tout paraissait vouloir incliner le cœur à la joie » $(T M, 87)$ qu'elle va plonger dans l'écriture de son roman-brouillon, objet transitionnel qu'elle traîne partout avec elle dans ses valises ( Depuis deux ans, même en n'ayant aucun espoir de travailler en voyage, je traînais avec moi ce qui devenait un énorme manuscrit. A cette époque, il devait bien compter huit à neuf cents pages » $T M, 88$ ).

Remarquons que cette Gaspésie, cet arrière-pays originel, est d'emblée présentée sous le signe du rêve : « Je rêvais de la Gaspésie sans la connaitre ; sans l'avoir jamais vue, je rêvais néanmoins d'elle comme on rêve sans l'avoir connue non plus de l'île de Pâques » (TM, 79). Mais ce lieu mythique, fabuleux, vaut moins ici par ses attraits extérieurs que parce qu'il se trouve en résonance intime avec l'épreuve du deuil : «Ainsi existent des pays qui correspondent exactement à nos rêves les moins explicables. A présent la mer apparaissait presque à chaque tournant, et à chaque tournant je prenais comme un plongeon dans cet infini » $(T M, 81)$. Même si la narratrice n'explicite pas le transfert qui se produit ici de la figure de sa mère à ce paysage marin auquel elle s'identifie si profondément, l'expérience de déjà-vu qu'elle dit éprouver en le voyant - « Je reconnaissais le pays jamais vu auparavant, tel que je m'en étais languie pourtant et l'avais souhaité du fond de mon cœur » $(T M, 81)$ - permet d'esquisser une telle hypothèse : le corps glorieux de la nature se substitue - transfiguré - tout du long ici à celui de la mère. Ce lieu physique avec ses bruits et ses odeurs, le cri des mouettes et le récitatif du vent, et surtout la présence de la mer sur fond d'infini a déjà tout pour mettre en scène une nature hantée par la question des origines. Ce paysage tellurique et primitif va en tout cas permettre à la narratrice de projeter sur lui sa nostalgie d'une relation symbiotique. Ce paysage lui offre déjà dans sa «surréalité 》 (un excès marque chacun de ses éléments : trop de soleil, trop de lumière, «agitation perpétuelle de l'air » $T M, 91)$ qui le déréalise, une exemplaire scène psychique pour déployer son deuil. Car ce cadre enchanteur, ce soleil étincelant surtout ne manquent pas de la précipiter dans le désespoir le plus noir, précisément parce que chaque paysage brillant, chaque objet éblouissant de lumière lui rappelle que, de ce monde, «maman n'était plus, [...] maman était exclue, [...] maman ne faisait plus partie » $(T M, 87):$ «Et toutes joies, toutes beautés pourtant proches, me parvenaient comme d'un monde lointain à jamais perdu que je ne connâ̂trais plus maintenant que par le souvenir $\gg(T M, 87)$. A cette étape du deuil de Gabrielle en Gaspésie, la présence de la mère subsiste, mais elle est diffuse et universelle, comme si «le sentiment du vide fai[sai]t écho à 
l'incapacité de penser la mère, à faire vivre l'enfance heureuse en soi (42)», comme le dit Mannoni à propos du travail de deuil chez Woolf. «Sa façon d'être là est de se trouver ailleurs : avec les hirondelles [Gabrielle, elle, ne cesse de suivre le vol des mouettes dans ces pages] ou plongée dans ses livres d'histoire. [...] [Elle] ne peut songer qu'à l'évasion dans l'imaginaire (43). » Ce sentiment de détresse est proche du « sentiment d'un impossible d'exister (44) » décrit par Freud dans son texte sur «l'éphémère »: «Le poète, écrit-il, admirait la beauté de la nature autour de nous, mais n'en éprouvait aucune joie. Il était troublé par la pensée que toute cette beauté était vouée à passer [...]. Comment retrouver alors le motif d'une joie créative - le pouvoir d'un temps de la création - alors que le renouvellement de la vie fait l'effet d'une dénégation (45) ? »

Or, c'est précisément pour retrouver cette joie créative qui lui est dérobée depuis la mort de sa mère, que la narratrice se met à écrire, sans savoir où elle va, ni pourquoi elle écrit, «ni même vers quoi [la] menait un si dur effort » $(T M, 89)$. Elle pressent qu'à défaut de cette écriture qui lui permet de retrouver le fil du temps - et éventuellement le temps de la création -, elle va sombrer dans «une mélancolie inguérissable (TM, 88) : l'écriture est le premier pas vers une restauration de soi, elle protège de la violence destructrice, elle remplit l'espace vide laissé par la disparition de la mère, qui a emporté une part de soi. «Je continuai donc sans plus savoir où me menait mon effort » $(T M, 89)$, répète la narratrice trouvant dans la dynamique esthétique un point d'ancrage la protégeant des fantasmes de destruction qui l'habitent à ce moment. « [...] Je n'étais pas encore en pleine possession de mon sujet. Il y aurait des pages à reprendre. Mais du moins la souffrance décrite paraissait plus vraie et plus impitoyable» $(T M, 89)$. D'ailleurs, maintenant que son travail est « sans but », il avance de mieux en mieux, et il est clair que l'écriture lui permet d'amorcer une essentielle conversion, opérant le transfert de la douleur dans une forme qui la contient. Et même si le récit reste «en partie romancé, c'est-à-dire transcendé » $(D E, 183)$, il parvient à dire l'essentielle vérité du deuil, à en représenter la séparation la plus déchirante et «la mystérieuse consolation à en naître » $(D E, 179)$. Entre sa mère disparue et elle dont l'identité vacille, il y a désormais cette ouvre qui s'élabore et dont elle s'occupe maternellement, comme un enfant, le manuscrit se nourrissant chaque jour davantage de sa peine qu'il transforme peu à peu en art. Comme dans le rapport d'entredeux et de va-et-vient qui soutenait toute la relation à la mère, une conversion se produit donc de la vie à l'art, de la souffrance «plus vraie» à sa transposition esthétique, qui opère en retour sur la vie en la rendant « plus impitoyable »- ce qui, incidemment, pourra surprendre : l'écriture, loin de 1957, p. 307, cité par Maud Mannoni, op. cit., p. 59. 
« guérir (46) » de manière trop idyllique, accentue au contraire l'écart entre les « histoires » et la « vraie vie ». « Du moment que je sortais de mes histoires je me sentais accablée. La vraie vie de plus en plus était pour moi dans ce que je me racontais ou racontais à quelqu'un qui pourrait un jour s'y reconnaître [...]. Cependant, dès que je sortais du travail, c'était pour me reprendre à souffrir d'être de ce monde » $(T M, 90)$.

Réfugiée dans sa chambre-coquille, construisant jour après jour une œuvre-embryon, avec sous les yeux le spectacle de la mer, ce repli sur soi, cette régression marquent aussi pour Gabrielle la création d'une île, d'un îlot en soi qui va enfin lui permettre de se séparer d'avec la mère, de trouver une manière de se débarrasser de «son obsession du manque de mère » grâce à son œuvre (47). C'est ce qui se fait jour dans l'ultime scène de ce récit autobiographique, qui se donne également comme la clausule et la clôture de toute l'œuvre.

Les éditeurs et la critique se sont empressés de lire ce texte de deuil sous le signe de la réconciliation (48). En guise de contrepoint, je voudrais pour ma part proposer une lecture un peu différente - une contre-lecture en fait - de ce texte et tout particulièrement de la dernière scène. Je m'appuierai sur certains passages de l'ouvrage de Maud Mannoni, Elles ne savent pas ce qu'elles disent, qui porte sur Virginia Woolf, et qui s'attache à l'un des aspects les plus prégnants de son cuvre, soit le deuil de la mère, et à cette « angoisse impensable liée à la perte de la relation archaïque de l'enfant à la mère $(49)$ », qui la réduisit finalement au silence. L'interprétation que propose Mannoni éclaire aussi ce qui est en jeu dans le texte de Gabrielle Roy. D'ailleurs, le détour n'est qu'apparent car il y a beaucoup d'affinités entre ces deux femmes écrivains, à commencer par leur manière de revendiquer leur « différence » malgré les interdits de leur époque, tout en demeurant assez ambivalentes par rapport à cette audace. Écrire pour Woolf comme pour Roy, «c'est remonter le temps, réparer les blessures, accéder à une plénitude (50) ». Mais revenons à la dernière séquence du Temps qui m'a manqué où, au cours d'un violent orage, et contre l'interdit de Mme Bertha de sortir, la narratrice, éprouvant « une étrange jubilation », met son ciré et s'éclipse «sans être remarquée » $(T M, 91)$. Battue par les vents, elle se fraie un chemin jusqu'à « la pointe de terre, de roc plutôt, s'avançant entre des falaises et de hauts récifs en pleine fureur de la mer» $(T M, 91)$ :

46 L'écriture n'est pas thérapeutique, mais avive au contraire la perte, la faisant travailler, non pas pour oublier la douleur donc, mais pour lui trouver une voie d'élaboration.

47 Ibid., p. 111. Maud Mannoni fait remarquer un processus analogue chez Woolf lorsqu'elle écrit La Prome. nade au phare: «Grâce à ce livre, elle n'entend plus sa voix, elle ne la voit plus, elle peut "laisser sa mère au repos". Cette mère, dans Entre les actes, réussit à ne plus occuper l'espace imaginaire de sa fille, sa présence est supprimée. Nous sommes d'une certaine façon conviés à assister à sa "seconde mort"."

48 «Ainsi, dans cet écrit ultime qu'elle n'a pas achevé mais auquel elle a tout de même réussi à conférer une beauté et une plénitude que seule la maîtrise parfaite de son art a pu rendre possibles, Gabrielle se réconcilie définitivement, pour ainsi dire, avec elle-même et avec son passé, rattrapant enfin, au moment de mourir, ce temps qui, jadis, lui a si cruellement manqué » ( « Avertissement des éditeurs". dans Le Temps qui m'a manqué, p. 10). 49 Maud Mannoni, op. cit., p. 49. C'est l'auteur qui souligne. 50 Ibid., p. 65. 
notons au passage à quel point le décor de cette scène est romantique, gothique même. Dans cet espace sauvage et déserté, entre ciel et terre, elle se réfugie dans « une sorte de grotte », « creusée à même la pierre », « toute proche de l'écume bondissante et des hurlements, mais bien protégée par une avancée du roc qui formait auvent » (TM, 92). Là, «blottie » dans ce trou de pierre, mais enroulée dans une couverture et «presque au chaud» (TM, 92), nouveau-né qui s'engendre elle-même, elle participe à la clameur des éléments, «l'eau, le tonnerre, les vagues, le vent [qui] jettent parfois comme de véhémente protestation à la face du ciel » $(T M, 92)$.

Sans être nulle part nommée, tout en restant de l'ordre du nonreprésentable, la présence de la mère est fortement sensible dans cette scène-symptôme où se trouve rejoué dans une spectaculaire mise en scène un traumatisme d'origine (51). Il n'est que trop tentant en effet de lire ce trou - qui soutient, soulignons-le, tout ce récit de deuil mais au-delà la totalité du récit autobiographique et peut-être même l'œuvre tout entière comme la représentation du corps maternel appartenant à un «temps d'avant la coupure du cordon ombilical (52) ». Comme le voit bien Régine Robin, « le trou est ambivalent. Source de joie, de maîtrise (on y est enfin chez soi), il est aussi le signe du malheur. Trou de l'identité, il connote à la fois l'enfermement essentialiste [...] et le refus d'une assignation quelle qu'elle soit, la possibilité d'échapper au destin (53) ». Autrement dit, le trou, la grotte dans laquelle se réfugie la narratrice est tout à la fois ce qui la sauve en l'abritant (elle insiste beaucoup sur le caractère somme toute douillet de sa retraite-nid) et ce qui la met en danger. Et cette grotte, c'est aussi, c'est surtout «la cachette de l'enfant, le lieu du rêve (54) ». Enfoncée dans ce coeur maternel, ce trou profond et noir où elle risque de se noyer, la narratrice émerge au terme de cette nuit initiatique «mystérieusement délivrée, comme si l'amertume (55) du moins m'avait été enlevée » $(T M, 92)$. Cette fin marque un commencement enfin redevenu possible ( $\ll$ Mais au fond, je suis toujours en peine de m'expliquer comment je sortis, cette nuit-là, sinon apaisée, du moins consentante à vivre en ce monde (56) »TM, 92).

51 Maud Mannoni rappelle à propos de la figure du traumatisme, « qu'on ne peut tout dire », que « ce travail de la vérité échoue néanmoins à révéler l'indicible » : «ll y a une part d'inassimilable dans le trauma, quéque chose qui, dans la réalité, demeure en souffrance » (op. cit., p. 35$)$.

52 J'emprunte cette idée à Régine Robin qui analyse la portée d'un tel « trou » dans l'œuvre de Romain Gary (Le Golem de l'écriture. De l'autofiction au Cybersoi, Montréal, XYZ éditeur, "Théorie et Littérature», 1997, pp. 113-114). 53 Ibid., p. 114. 54 lbid., p. 113.

55 Il est intéressant de souligner dans ce mot la mère/amère, avec laquelle la fille, dans sa demande insatiable de mère, a encore des comptes à régler, elle qui lui est restée depuis sa mort « en travers » (j'emprunte cette expression à Maud Mannoni, op. cit., p. 168).

56 Ces derniers mots ne sont pas les tout derniers, car les éditeurs du manuscrit ajoutent en note cette phrase, sur une feuille volante insérée à la dernière page du cahier : «Je n'ai donc pas souvent rencontré de douleur qui $n$ 'ait pas laissé au moins une toute petite part à quelque joie, de même que je n' as pas tencontré souvent de joie si grande quelle n'ait laissé entrer dans la place quelque sentiment de douleur » (TM, 102). Cette phrase révélatrice jusque dans sa construction et son rythme de l'oscillation entre détresse et enchantement, reconduit ultimement le mouvement de conversion et de reconversion - - de la joie à la douleur, de la douleur à la joie à l'infini. 
Toutefois, contrairement à la réconciliation sur laquelle on a beaucoup insisté, sans doute parce qu'elle était rassurante, ce qui me frappe dans cette scène c'est la manière dont Gabrielle Roy parvient à y exprimer sa rage à travers le déchaînement des éléments, c'est la manière dont elle laisse exploser, en la projetant sur l'Autre scène, les mots retrouvés dans la violence, qui seulement à ce moment auront pour effet une force de «délivrance ». On sent bien que la parole à l'œuvre ici est à la fois véridique et mensongère, que l'imaginaire emprunte un déguisement pour s'approcher d'une vérité impossible à dire, d'une angoisse impensable liée au tabou de la mère, toute référence à la mère mauvaise et à la haine inconsciente de la mère se trouvant interdite (dans la scène des adieux de La Détresse... où il était enfin question de la «perdre de vue», c'était bien cette haine inconsciente qui affleurait de manière détournée à travers l'affect de la honte). Ainsi, si cette scène est si forte, c'est moins par sa résolution apparemment heureuse, que par l'excès, l'effroi, la violence destructrice de cette ultime confrontation avec l'image de la mère. Gabrielle Roy aurait pu, cédant comme Woolf à ce "grand désir d'eau », compenser le manque en se jetant dans les flots. Elle choisit plutôt de (re)naître, «transie, mouillée jusqu'aux os », « endolorie de la tête aux pieds » $(T M, 92)$, récupérant sa voix qui «s'était égarée dans celle de sa mère (57) » : «Il me semblait que criaient pour moi, ou avec moi qui était silencieuse, les vents, les vagues, quelques goélands réveillés et en déroute, et que tous ensemble, les vagues, l'écume, les vents, les oiseaux dérangés plaignaient à n'en plus finir la douleur du monde » $(T M, 92)$. Cette scène est donc bien plutôt l'envers de la réconciliation : c'est une scène de radicale séparation où en «se dégage[ant] de la morte», il s'agit d'une tentative de se récupérer comme sujet (58). D'ailleurs, on pourrait aussi dire que le manuscrit qui absorbe désormais la narratrice créera enfin, tel un objet transitionnel, une aire de jeu, « un espace potentiel » tel que le décrit Winnicott, entre elle et sa mère, «entre le subjectif et l'objectif, entre le sujet et son environnement (59) ». Loin d'être seulement un «trophée » ou une «médaille » qu'elle serait allée « au loin chercher [comme] ce qu'il y avait de meilleur » $(T M, 13)$ pour le rapporter à la mère («Et elle en serait à jamais réjouie » $T M, 13)$, l'œuvre est aussi posée comme un rempart contre la mère, «qui lui permet de la tenir à distance. C'est un moyen de se protéger de [ses] exigences (60) ».

On aperçoit mieux comment cette dernière scène nous fait assister, sur le plan du fantasme, à l'impensable du travail de deuil : à savoir non pas seulement comment garder en soi la figure disparue, en une infinie indistinction et dissolution fantomatique de sa voix en soi, mais aussi comment se débarrasser, oublier et dire adieu à la figure tant aimée. Dans cette ultime scène de son autobiographie, Gabrielle Roy nous fait assister à la seconde 
mort de sa mère en quelque sorte, prix de sa (re)naissance en écrivain. L'indicible auquel elle s'attache dans ces ultimes pages n'a rien d'idyllique, il est imprégné de l'ambivalence des sentiments d'amour et de haine, inextricablement mêlés. C'est bien un chant d'amour qu'elle nous fait entendre, mais «sur fond de violence sourde, celle de la haine qui gît au cœur de l'amour (61) ». Et de cette épreuve formatrice du deuil, elle sort profondément « remaniée », comme le texte qu'elle reprend et réélabore : il n'est que de mesurer le déplacement qui s'est opéré de la figure de la mère vers qui, disait-elle au début du récit, « je n'avais cessé d'aller [...], inconsciemment ou non (62) 》 $(T M, 22)$, à ce lecteur anonyme, ce « quelqu'un qui pourrait un jour [se] reconnaître [dans ses histoires], et alors peut-être, en silence, nous rejoindrions-nous, moi qui marchais seule et cet inconnu dans l'avenir, quelque part arrêté à attendre il ne savait quoi » (TM, 90).

On l'entrevoit à partir de cette lecture des textes autobiographiques posthumes de Gabrielle Roy : la pratique de l'autobiographie aura bien été pour elle le lieu d'une complexe naissance, engendrement de soi comme sujet dans et grâce à l'écriture, passant tour à tour par la fictionnalisation, le dévoilement et la perte de soi. On ne saurait douter que l'écrivain a porté aussi loin qu'elle l'a pu la «construction de soi, voire cette fictivisation de soi qui est l'essence du projet autobiographique (63), et on doit reconnaitre l'étonnante modernité de sa démarche, qui fait trembler les partages traditionnels entre fiction et autobiographie. Il est difficile de dire en quoi un exercice aussi remarquable de l'écriture de soi relèverait encore d'une «spécificité » de la littérature québécoise - l'entreprise gagnerait davantage à être lue à une autre hauteur, celle de modèles intertextuels finement convoqués ici comme ceux de Rousseau ou de Proust, ou encore de venues à l'écriture comme celles de Colette ou de Virginia Woolf (64) -, mais il est clair, pour faire écho à une idée chère à André Belleau qui pensait qu'une littérature comme la nôtre était moins assurée d'exister par ses œuvres de fiction que par les essais et réflexions qu'elle parviendrait à nourrir, que l'autobiographie de Roy devrait elle aussi compter comme œuvre pensante fondatrice, lieu de réflexion où est saisi le sujet même de la littérature. Une telle entreprise fait en tout cas singulièrement exister la littérature québécoise, en interrogeant de manière aussi profonde les transferts à l'œuvre entre la vie et l'écriture, et en croyant tout simplement à ce point à la force transformante de la littérature.

61 Ibid., p. 62.

62 « Mais bientôt, à tant penser à elle, je m'apaisais. C'était comme si elle m'eut rejointe pour faire route avec moi puisque nous allions dans le méme sens $[\ldots] »(T M, 23)$. Il est révélateur de remarquer qu'elle avance paralièlement à la mère (« dans le mêrne sens»), côte à côte, signe de la non-rencontre même, alors qu'avec le lecteur futur, les lignes de leur trajectoire respective pourront se croiser en un point inconnu. 